\title{
Oncocitoma em carúncula: relato de caso
}

\author{
Oncocytoma of the caruncle: a case report \\ Bruno Hirt ${ }^{1}$ (D), Marcos Bortoluzzi Worma² (), Graziela Junges Crescente Rastelli ${ }^{3}$ (), Rodrigo Beraldi Kormann ${ }^{4}$ (1) \\ ${ }^{1}$ Curso de Especialização em Oftalmologia, Hospital de Olhos do Paraná, Curitiba, PR, Brasil. \\ ${ }^{2}$ Curso de Medicina Universidade Positivo, Curitiba, PR, Brasil. \\ ${ }^{3}$ Departamento de Medicina, Faculdade Evangélica Mackenzie do Paraná, Curitiba PR, Brasil. \\ ${ }^{4}$ Departamento de Oftalmologia, Hospital de Olhos do Paraná, Curitiba, PR, Brasil.
}

Oncocitoma/diagnóstico: Adenoma oxífilo/diagnóstico; Anexos oculares; Oftalmopatias; Carúncula; Neoplasias oculares

Keywords:

Oncocytoma/diagnosis; Adenoma, oxyphilic/diagnosis; Ocular adnexa; Eye diseases; Caruncle; Eye neoplasms

Recebido: 9/7/2020

Aceito: 3/4/2021

Autor correspondente: Bruno Hirt

Rua Prof. Pedro Viriato Parigot de Souza, 3155. Campo Comprido, Curitiba, PR 81200-452, Brasil.

E-mail: brunohirt.med@gmail.com.

Instituição:

Hospital de Olhos do Paraná, Curitiba

PR, Brasil.

Fonte de auxílio à pesquisa: não financiado.

Conflitos de interesse: os autores declaram que não há conflitos de interesses.

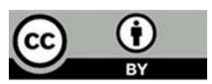

Copyright (C2021

\section{RESUMO}

O oncocitoma é um tipo de neoplasia rara na prática clínica e descrito na literatura, principalmente quando localizado no olho. Quando localizado nos anexos oculares, é mais frequentemente na carúncula. Analisou-se o caso de uma paciente de 74 anos, caucasiana, que relatou desconforto visual no olho esquerdo, e cujo exame físico mostrou lesão tumoral na carúncula esquerda, com volume moderado, presença de neovascularização e secreção excessiva. A excisão cirúrgica da lesão foi realizada sob sedação, e a peça foi enviada para avaliação anatomopatológica. A lesão foi diagnosticada histologicamente como oncocitoma, sem malignidade, e a paciente não apresentou recidiva após o procedimento. Embora raro, esse tumor deve ser reconhecido pelos oftalmologistas, devido ao risco já relatado de desenvolvimento de adenocarcinoma.

\section{ABSTRACT}

Oncocytoma is a neoplasm rarely observed in clinical practice and reported in the literature, especially when located in the eye. When described in the ocular adnexa, it is most often located in the caruncle. The case of a 74-year-old Caucasian female patient is reported. She complained of visual discomfort in the left eye, and physical examination showed a tumoral lesion in the left caruncle, of moderate volume, presence of neovascularization, and excessive secretion. Surgical excision of the lesion was performed under sedation, and the specimen was sent for pathological examination. The lesion was histologically diagnosed as oncocytoma with no malignancy, and the patient presented no recurrence after the procedure. Although rare, this tumor must be recognized by ophthalmologists due to the risk of developing adenocarcinoma, as already reported. 


\section{INTRODUÇÃO}

O oncocitoma é um tipo de neoplasia descrita pela primeira vez nos anexos oculares de Radnót, em 1941.(1) Esse tipo de tumor é raramente encontrado na prática clínica e na literatura, principalmente quando localizado no olho, sendo mais comum nos rins, nas glândulas salivares, na tireoide e na paratireoide. ${ }^{(2)}$ Entre os anexos oculares, é mais frequentemente encontrado na carúncula, seguida da conjuntiva, do saco lacrimal e da glândula lacrimal. (3-5) $^{(3-1}$

Os oncocitomas são histologicamente derivados de oncócitos, que são células originadas da degeneração metaplásica no tecido epitelial secretor. Sua característica definidora é uma grande quantidade de citoplasma eosinofílico de aspecto homogêneo, repleto de mitocôndrias defeituosas, enzimas oxidativas e adenosina trifosfatase. . $^{(1,6)}$

Morfologicamente, eles geralmente se apresentam como massas sésseis, bem delineadas e azul-escuras, com cistos intralesionais e penetração até a camada subepitelial. A vascularização intrínseca também é comum. ${ }^{(5)} \mathrm{A}$ malignidade é extremamente rara, mas alguns casos foram relatados. ${ }^{(3)}$

Clinicamente, é frequentemente confundido com hemangiomas, nevos e cistos. ${ }^{(7)}$ Neste relato de caso, são abordadas informações como sintomas, diagnóstico e tratamento. A coleta e a avaliação de informações protegidas de saúde do paciente neste estudo aderiram aos princípios da Declaração de Helsinque.

\section{RELATO DO CASO}

Paciente do sexo feminino, 74 anos, caucasiana, compareceu a uma consulta oftalmológica no Hospital de Olhos do Paraná, em Curitiba (PR), em novembro de 2019, relatando desconforto visual, sensação de corpo estranho e secreção serosa abundante no olho esquerdo. Não foi encontrado histórico médico familiar ou pregresso sobre lesões oftálmicas.

O exame físico da paciente mostrou, além de tempo de ruptura do filme lacrimal reduzido, uma lesão tumoral na carúncula do olho esquerdo de volume moderado, com presença de neovascularização e secreção excessiva (Figura 1).

Uma vez que a cirurgia foi planejada com a aprovação da paciente, a excisão cirúrgica da lesão foi realizada sob sedação, sendo encaminhada para exame anatomopatológico.

O resultado anatomopatológico revelou segmento de mucosa conjuntival removido da carúncula esquerda medindo 6x5x3mm, com diagnóstico histológico de oncocitoma na ausência de malignidade (Figura 2).

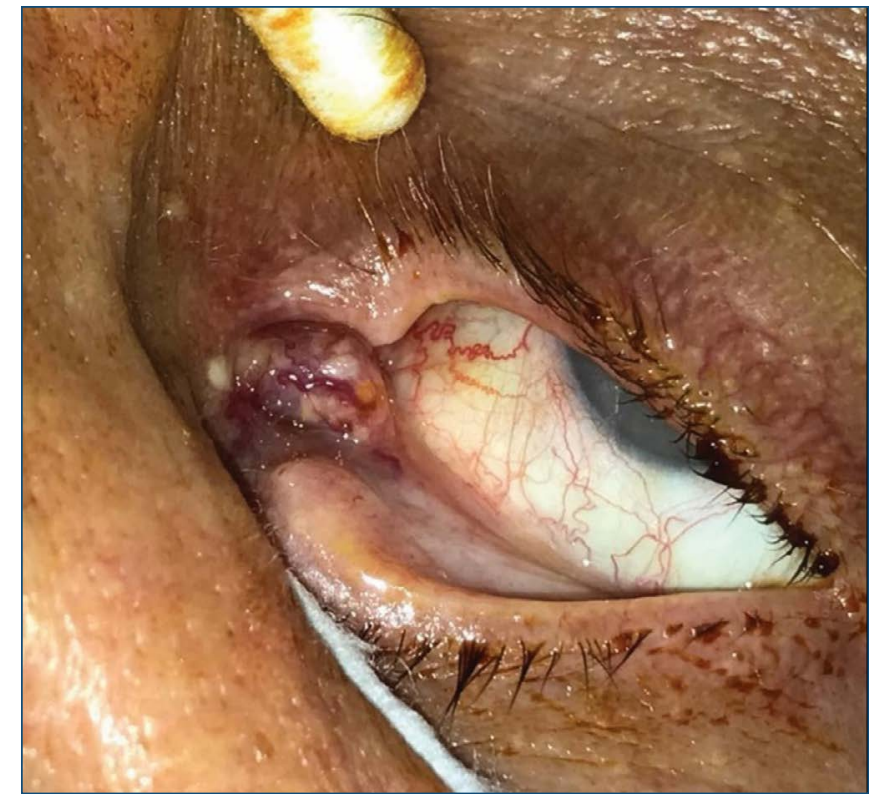

Figura 1. Foto pré-operatória do oncocitoma em carúncula do olho esquerdo de volume moderado e com presença de neovascularização.

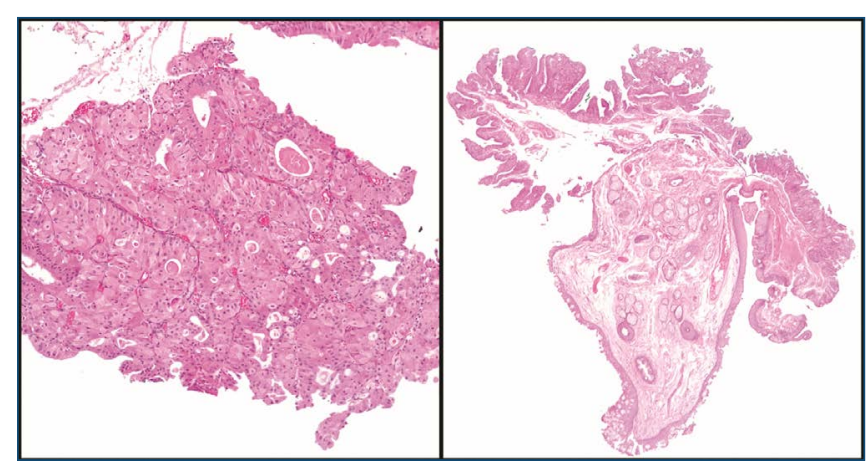

Figura 2. Corte histológico corado pela técnica hematoxilina-eosina evidenciando neoplasia benigna composta exclusivamente por células oxifílicas, caracterizadas por células grandes com núcleos redondos e abundante citoplasma acidofílico e granular. Ultraestruturalmente, sabe-se que o citoplasma está repleto de mitocôndrias. Não foram observadas figuras de mitose, atipias citológicas nem focos de necrose tumoral.

A paciente retornou para avaliação pós-operatória no primeiro, sétimo e $30^{\circ}$ dia após a cirurgia. Não houve recorrência ou outros sintomas.

\section{DISCUSSÃO}

Lesões oncocíticas são formadas por oncócitos, grandes células epiteliais poligonais que apresentam um citoplasma altamente eosinofílico repleto de mitocôndrias defeituosas. Supõe-se que, nas células envelhecidas, a perda de mecanismos de proteção do DNA contra o estresse oxidativo leve a esse acúmulo. Pleomorfismo, mitoses e necrose tumoral geralmente estão ausentes. $(1,6,7)$ O crescimento descontrolado dessas células pode 
caracterizar um oncocitoma, o qual pode ser encontrado em vários órgãos, bem como nos anexos oculares, nos quais geralmente acomete a carúncula, podendo, porém, ocorrer em pálpebras, conjuntiva, saco lacrimal e glândula lacrimal. ${ }^{(2,5)}$

Oncocitomas localizados em carúncula já foram descritos nas últimas décadas, ${ }^{(2,4,4,5,7-10)}$ porém continuam sendo raros de serem encontrados na prática clínica diária, visto que representam menos de 1\% de todas as amostras patológicas relacionadas a anexos oculares. ${ }^{(5)}$ Entretanto, são mais prevalentes em pacientes idosos e do sexo feminino, assim como o caso descrito no presente trabalho, devendo ser sempre considerado um diagnóstico diferencial em pacientes com essas características. . $^{(2-4,8,11)}$

Alguns estudos afirmaram que o principal local de envolvimento de uma lesão oncocítica era a carúncula. $(2,4,5)$ Além disso, sabe-se que as lesões tendem a medir menos de $1 \mathrm{~cm}$, ${ }^{(4)}$ e a literatura já estabeleceu um tamanho médio de 4X4X3 mm em uma série de $\operatorname{casos}^{(5)}$ - dimensões muito semelhantes às da paciente estudada.

A história natural da doença é caracterizada como uma massa indolor, com crescimento lento e geralmente assintomático. ${ }^{(4,9,10)}$ Sua remoção ocorre principalmente por razões diagnósticas, por meio de biópsia do tecido acometido, cosméticas ou quando, além dos já mencionados, gera algum desconforto para o paciente, como aconteceu neste caso.
Embora raro, esse tumor deve ser reconhecido pelos oftalmologistas devido ao desenvolvimento já relatado de adenocarcinoma. ${ }^{(3)} \mathrm{A}$ excisão cirúrgica continua sendo o método de escolha para tais casos, pois as recidivas são extremamente raras após a remoção completa da lesão. ${ }^{(3-5,11)}$

\section{REFERÊNCIAS}

1. Radnót M. [Aus Onkocyten bestehende adenomartige Hyperplasie in der Tränensackwand]. Ophthalmologica. 1941;101(2):95-100. German.

2. Østergaard J, Prause JU, Heegaard S. Oncocytic lesions of the ophthalmic region: a clinicopathological study with emphasis on cytokeratin expression. Acta Ophthalmol. 2011;89(3):263-7.

3. Biggs SL, Font RL. Oncocytic lesions of the caruncle and other ocular adnexa. Arch Ophthalmol. 1977;95(3):474-8.

4. Mulay K, Rasmussen PK, Aggarwal E, Honavar SG, Heegaard S Accessory lacrimal gland tumours of the eye region. Acta Ophthalmol. 2018;96(7):e772-5.

5. Say EA, Shields CL, Bianciotto C, Eagle RC Jr, Shields JA. Oncocytic lesions (oncocytoma) of the ocular adnexa: report of 15 cases and review of literature. Ophthal Plast Reconstr Surg. 2012;28(1):14-21.

6. Hamperl H. Benign and malignant oncocytoma. Cancer. 1962;15:1019-27

7. Jones EK, Lozeau DF, Lee JB. Oncocytoma of the lacrimal caruncle and its relevancy to dermatopathology. J Cutan Pathol. 2016;43(3):298-300.

8. Mitra S, Lath K, Samanta R, Saikia UN. Caruncular oncocytoma: report of two cases with review of literature. Indian Dermatol Online J. 2018;9(5):324-7.

9. García CA, Melo-Uribe MA, Díaz JA. [Oncocytoma of the lacrimal caruncle]. Actas Dermosifiliogr. 2008;99(7):581-2. Spanish.

10. Souza Filho JP, Vianna RN, Coutinho AB, Arthurs B, Burnier MN Jr. Oncocytoma of the caruncle: a clinicopathologic case report. Int Ophthalmol. 2004;25(5-6):321-3.

11. Shields CL, Shields JA, Arbizo V, Augsburger JJ. Oncocytoma of the caruncle. Am J Ophthalmol. 1986;102(3):315-9. 